\title{
Incidencia de la retro-innovación en educación superior. Radio y televisión como herramientas complementarias en el uso del modelo de enseñanza conocido como aprendizaje invertido
}

\author{
Santiago Pozo-Sánchez, Jesús López-Belmonte*, Arturo Fuentes-Cabrera y Antonio-José Moreno-Guerrero \\ Facultad de Educación, Economía y Tecnología, Dpto. de Didáctica y Organización Escolar, Universidad de Granada, \\ Ceuta 51001, Cortadura del Valle, Granada-España. (correo-e: santiagopozosanchez@correo.ugr.es; \\ jesuslopez@ugr.es; arturofuentes@ugr.es; ajmoreno@ugr.es)
}

* Autor a quien debe ser dirigida la correspondencia.

Recibido Nov. 25, 2019; Aceptado Ene. 20, 2020; Versión final Ene. 30, 2020, Publicado Jun. 2020

\begin{abstract}
Resumen
El estudio se centra en analizar el alcance de dos enfoques metodológicos para comprobar su incidencia en diversos indicadores académicos. El diseño experimental es de carácter cuantitativo, descriptivo y correlacional. El estudio incluye un grupo control y un grupo experimental en una muestra de 60 estudiantes universitarios españoles. El grupo control ha seguido una metodología formativa de índole tradicional, sin utilización de recursos tecnológicos. El grupo experimental ha desarrollado una acción pedagógica mediante aprendizaje invertido (flipped learning) apoyado en recursos clásicos como la radio y televisión. Para la recogida de datos se ha empleado un cuestionario que ha sido diseñado y validado para esta investigación. Los resultados reflejan que el grupo experimental ha obtenido mejores valoraciones en la colaboración, motivación, rol activo, interacción, competencia digital y logros de aprendizaje, sobre el grupo control. Se concluye que la combinación de flipped learning junto con medios retro innovadores resulta eficaz en la mejora de indicadores académicos en educación superior.
\end{abstract}

Palabras clave: tecnología educativa; innovación formativa; aprendizaje invertido; retroinnovación; contraste metodológico

\section{Incidence of retro-innovation in higher education. Radio and television as complementary tools when using the educational model known as flipped learning}

\begin{abstract}
This study focuses on analyzing the scope of two methodological approaches to verify their impact on various academic indicators. The experimental design is quantitative, descriptive, and of correlational character. The study is composed of a control and an experimental group from a sample of 60 Spanish university students. The control group followed a traditional training methodology without the use of technological resources. The experimental group developed a pedagogical action through flipped learning that was supported with classical resources such as radio and television. A questionnaire was designed and validated for data collection. The results showed that the experimental group obtained better evaluations than the control group in the following indicators: collaboration, motivation, active role, interaction, digital competence, and learning achievements. It is concluded that combining flipped learning with retro-innovative media is effective for improving academic indicators in higher education.
\end{abstract}

Keywords: educative technology; formative innovation; flipped learning; retroinnovation; methodological contrast 


\section{INTRODUCCIÓN}

A día de hoy, el gran avance acaecido en el ámbito tecnológico está generando nuevas dinámicas y tendencias en la sociedad que determinan nuestra forma de vivir (Mehandru y Koenig, 2019). El campo de la educación no es ajeno a ello, haciendo -cada vez más- un mayor uso de ella en los procesos formativos (Area et al., 2016). La inclusión de la tecnología en los actos pedagógicos necesita de una preparación y cualificación por parte de los docentes para poder llevar -con un mínimo de eficacia- su aplicación en el aula mediante el uso de metodologías activas (Rodríguez et al., 2019). Esta transformación de la educación en la era digital (Villareal et al., 2019) ha provocado que el rol de los principales actores educativos se intercambie, pasando el docente a tener el papel de guía y los estudiantes de precursores de sus conocimientos (Cabero, 2017). En otras palabras, el nuevo protagonista del aprendizaje es el alumno, quien construye su propio conocimiento, recibiendo en determinados momentos las orientaciones necesarias por parte de los docentes para que adquiera una formación adecuada de acuerdo a los nuevos paradigmas educativos que transcurren en la actual sociedad del conocimiento, caracterizada por el rápido y fácil acceso a la información (Garrote et al., 2016).

Los recursos innovadores generan nuevos paradigmas socioeducativos, donde los formadores tratan de alcanzar la motivación e implicación de los estudiantes, además de activar los procesos de enseñanza y aprendizaje, promoviendo así nuevos escenarios pedagógicos (Cabero y Barroso, 2018). Por ello, en la educación del nuevo milenio el docente requiere del desarrollo de una serie de habilidades y destrezas que le permitan hacer uso eficiente de la tecnología educativa. Entre dichas habilidades se encuentra la competencia digital, convirtiéndose en un elemento crucial para actuar desde el nuevo paradigma educativo socio-constructivista (Pascual et al., 2019). Esta competencia abarca una serie de áreas en las que el profesorado debe mostrarse competente: Información y alfabetización informacional; Comunicación y Colaboración, Creación de contenidos digitales; Seguridad; Resolución de problemas (Fuentes et al., 2019).

Además de la competencia digital docente, los formadores necesitan otras habilidades para llegar a la excelencia en sus métodos pedagógicos, siendo una de ellas la creatividad, la cual promueve la aparición de la retroinnovación como recurso pedagógico (Soberanes et al., 2017). Diversos autores destacan que el uso de recursos tecnológicos tradicionales -tales como la televisión o la radio- con fines educativos son valorados positivamente por los propios estudiantes (Rodas y Aveiga, 2017). Ello les permite crear sus propios conocimientos con altas dosis de participación e implicación, generando así nuevos escenarios de aprendizaje en el que se intercambian ideas y valoraciones críticas entre los propios estudiantes. Todo ello deriva en el fomento de la comunicación, participación e interacción entre los diferentes agentes que articulan el proceso formativo (Fuentes y López, 2018).

Otros autores son más explícitos y determinan que la televisión y la radio desde una vertiente didáctica promueven el aprendizaje por competencias en los estudiantes, consiguiendo desarrollar -además-actitudes críticas, al ser selectivos en la elección de la información, gracias al trabajo colectivo, la dialéctica (Martín et al., 2018), la participación, la reflexión, la coherencia, la sociabilización y la atención (Cabero, 2017). Un aspecto a tener en cuenta son las características de los dispositivos móviles actuales, que permiten generar nuevas experiencias formativas, gracias a la gran cantidad de recursos y herramientas que presentan (Soberanes et al., 2017). Asimismo, estos dispositivos móviles se pueden combinar fácilmente con elementos retroinnovadores como los citados anteriormente, permitiendo globalizar los procesos de enseñanza y aprendizaje, ampliar los espacios, medios y enfoques formativos, promover el trabajo colaborativo y la interacción entre docente-dicente (Basantes et al., 2017).

Entre los nuevos enfoques tecnopedagógicos, se sitúa el modelo flipped learning, concebido como una metodología de enseñanza y aprendizaje mixta (Froehlich, 2018). Esta innovación tiene sus inicios en 2012, surgiendo de la necesidad de atender a aquellos estudiantes que -por diversos motivos- no podían asistir al aula. Para que pudieran seguir el proceso de enseñanza, dos expertos en el campo de la tecnología educativa como Jonathan Bergmann y Aaron Sams confeccionaron materiales audiovisuales para que los discentes lo visualizaran en cualquier lugar y dispositivo con acceso a Internet, consiguiendo la ubicuidad en la acción formativa y que todos los estudiantes pudieran acceder a los contenidos fuera del entorno escolar para posteriormente- destinar el tiempo en el aula para ampliar los contenidos y llevar a cabo un aprendizaje basado en la práctica (Bergmann y Sams, 2012). Este hecho no pasó desapercibido para otros docentes, haciendo uso de este método de enseñanza, permitiendo así su extensión en los procesos pedagógicos (He et al., 2016).

Su aplicación metodológica se centra principalmente en alternar los espacios y momentos de aprendizaje (EI Miedany, 2019). En el desarrollo de las sesiones presenciales, los estudiantes dedican su tiempo para actuaciones puramente prácticas, dinámicas y resolutivas, con lo que se produce un incremento de la interación de los discentes con sus semejantes, con el profesorado y con los propios contenidos (Boelens et 
al., 2018; Long et al., 2017), mientras que fuera del entorno escolar -en cualquier lugar y momento- se visualizan los contenidos audiovisuales de carácter instructivo que han sido generados por los docentes, siendo para ello fundamental un adecuado nivel de competencia digital en sus distintas áreas y en especial a la concerniente a la creación de contenidos, ya que la calidad formativa del recurso generado debe ser pertinente para originar en el alumno una inquietud por aprender (López, Pozo, Fuentes y López, 2019).

Este planteamiento pedagógico permite una mayor interrelación entre los agentes implicados en el proceso de enseñanza y aprendizaje (Hwang et al., 2015), una mejor autorregulación del aprendizaje (Tourón y Santiago, 2015), un aumento de la motivación, participación, colaboración (Tse et al., 2019) y socialización de los estudiantes (MacLeod et al., 2017), repercutiendo todo ello en la mejora de los resultados académicos (Hinojo et al., 2018). Esto conduce a valoraciones positivas de los estudiantes hacia esta puesta metodológica ya que ven en este enfoque metodológico una nueva forma de exportar el aprendizaje a otros ámbitos que traspasan las barreras físicas de los centros educativos (Lee et al., 2018).

Mostradas las evidencias en el ámbito científico acerca del uso del flipped learning y de los medios audiovisuales tradicionales, surge el presente estudio desarrollado en el Grado en Educación Social de la Facultad de Educación, Economía y Tecnología de Ceuta (España) y materializado en la asignatura "Implicación y Participación ciudadana ante la exclusión Social. Las escuelas de familia". Los objetivos planteados en la mencionada asignatura se han trabajado a partir de dos modalidades de enseñanza y aprendizaje y -por consiguiente- por dos subgrupos muestrales independientes: uno que ha seguido una metodología tradicional y otro que ha sido sometido a una experiencia innovadora avalada por el Programa de Apoyo a la Docencia de la Universidad de Granada.

Por todo lo expuesto, el objetivo de la presente investigación -enmarcado en la experiencia desarrolladaconsiste en cuantificar, analizar y comparar los resultados obtenidos en un grupo que sigue un método de enseñanza y aprendizaje tradicional con respecto a otro grupo que sigue un modelo pedagógico retroinnovador en el que la radio y la televisión se constituyen como canales de aprendizaje para la aplicación del flipped learning.

\section{METODOLOGIA}

La experiencia ha consistido en la implementación del flipped learning como metodología vehicular de la asignatura, procediéndose a la creación por parte del alumnado de diversos documentales sobre la exclusión social registrada en la Ciudad Autónoma de Ceuta empleando la radio y la televisión como herramientas protagonistas y presentando los resultados en tertulias radiofónicas semanales (Fig 1). Mediante esta experiencia desarrollada, el alumnado experimentó en primera persona y desde distintos lugares de la Ciudad anteriormente citada el concepto de exclusión social, a través de los relatos de los ciudadanos y de las propias entrevistas generadas en diversos ambientes sociales caracterizados por la marginación que sufren determinados colectivos. Los alumnos se encargaron de captar imágenes y audios con la finalidad de generar a posteriori unos contenidos que permitieran el diálogo y el debate constructivo para alcanzar la asimilación y difusión del conocimiento. Los contenidos multimedia que generaron, formaron parte de una exposición colectiva desarrollada en unas Jornadas que se centraron en el objeto de estudio de este trabajo, la exclusión social en la Ciudad Autónoma de Ceuta, transfronteriza y caracterizada por altos índices de marginación y falta de inclusión social.

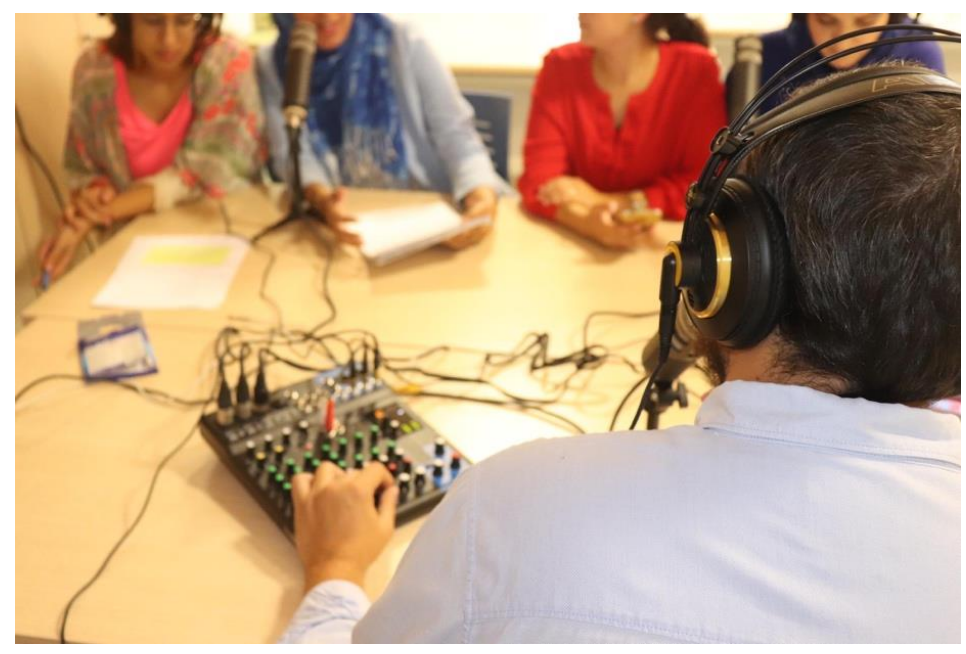

Fig. 1: Puesta en práctica de la experiencia. 


\section{Procedimiento}

Para poder alcanzar los objetivos propuestos en el presente estudio y en base a las recomendaciones de los especialistas (McMillan y Schumacher, 2005), se ha optado por llevar a cabo un método cuantitativo, descriptivo y correlacional fundamentado en un diseño experimental (Rodríguez, 2011) que emplea dos grupos de estudio, uno de control y otro experimental. El grupo control ha seguido una metodología tradicional de enseñanza y aprendizaje mientras que al grupo experimental ha seguido una experiencia retroinnovadora con flipped learning, radio y televisión. Tras la aplicación de ambas metodologías se procedió a la realización de un análisis para poder medir los resultados que se produjeron y su variabilidad con respecto a los obtenidos por el grupo control.

\section{Muestra}

La muestra de participantes se compone de un conjunto de alumnos $(n=60)$ de la Facultad de Educación, Economía y Tecnología de Ceuta que cursa la asignatura "Implicación y Participación ciudadana ante la exclusión Social. Las escuelas de familia", dentro del Grado en Educación Social. La elección de la muestra se llevó a cabo mediante un muestreo dirigido o intencionado, decisión tomada por la facilidad para poder acceder a los sujetos (Hernández et al., 2014). De esta forma, existe una distribución simétrica dentro de la muestra en lo concerniente a la subdivisión entre el número de participantes que integran el grupo control y el grupo experimental, tal y como se observa en la tabla 1.

También existe una distribución equitativa en cuanto al género de la muestra, con una leve predominancia del género femenino. A pesar de ello, el análisis estadístico llevado a cabo a partir de la prueba Chi-cuadrado no constató evidencias estadísticamente significativas en cuanto a un posible desequilibrio entre el grupo y el género $\left(X^{2}(1)=0,07 ; p=0,795\right)$.

Tabla 1: Participantes del estudio

\begin{tabular}{|l|c|c|c|c|c|c|}
\hline \multirow{2}{*}{ Grupo } & \multicolumn{2}{|c|}{ Masculino } & \multicolumn{2}{c|}{ Femenino } & \multicolumn{2}{c|}{ Total } \\
\cline { 2 - 7 } & $\mathrm{n}$ & $\%$ & $\mathrm{n}$ & $\%$ & $\mathrm{n}$ & $\%$ \\
\hline Experimental & 14 & 41.4 & 16 & 58.6 & 30 & 50 \\
\hline Control & 13 & 31 & 17 & 69 & 30 & 50 \\
\hline Total & 27 & 36.2 & 33 & 63.8 & 60 & 100 \\
\hline
\end{tabular}

\section{Cuestionario}

Con respecto al instrumento para la recogida de datos, se empleó un cuestionario de naturaleza ad hoc creado con base en los requerimientos específicos del estudio conformado por un total de 26 ítems y 3 dimensiones (1-Sociodemográfica; 2-Contribución al aprendizaje; 3-Pertinencia metodológica). El formato de respuesta se constituye de forma combinada entre respuesta abierta, respuesta múltiple y respuesta tipo Likert con escala de cuatro puntos (siendo 1 el valor más negativo y 4 el más positivo).

Dicho cuestionario fue sometido a un proceso de validación. En primer lugar, se realizó una validación de naturaleza cualitativa mediante juicio de expertos (Escobar y Cuervo, 2008) constituido por ocho Doctores especialistas en el campo de la Educación Social e integrantes de un compendio de universidades españolas. Estos expertos revelaron una opinión favorable del instrumento $(M=4.76$; $D T=0.71 ; \operatorname{mí}=1 ; \max =6)$ y ofrecieron a los investigadores distintas pautas para retroalimentar la herramienta y conseguir su optimización. Estas se focalizaron en la agrupación de determinadas cuestiones para reducir el número de ítems y en la modificación de la redacción de ciertos elementos para reducir el sesgo de incomprensión a la hora de cumplimentar el cuestionario por parte de los participantes. Del mismo modo, para conocer a nivel estadístico los índices de pertinencia y concordancia entre las valoraciones ofrecidas por los jueces se realizaron las pruebas Kappa de Fleiss y W de Kendall, resultando ambas adecuadas ( $K=0.84 ; \mathrm{W}=0.86)$.

\section{Validación}

A continuación, se inició la validación de corte cuantitativo por medio de un análisis factorial exploratorio, siguiendo el método de componentes principales y una rotación de tipo varimax. Las pruebas efectuadas en esta validación reflejaron dependencia entre las variables establecidas (Bartlett=2347.74; $p<0.001$ ) y una correcta adecuación de la muestra (Kaiser-Meyer-Olkin=0.87). En última instancia, se procedió al cálculo de diferentes estadísticos para obtener la fiabilidad del cuestionario. Se utilizó el alfa $(\alpha)$ de Cronbach, la fiabilidad compuesta $(F C)$ y la varianza media extractada (VME), los cuales reflejaron valores adecuados $(\alpha=0.87$; $\mathrm{FC}=0.84 ; \mathrm{VME}=0.85$ ) que permiten decretar una fiabilidad pertinente en el instrumento diseñado. 
Las variables formuladas para llevar a cabo el análisis comparativo entre ambos grupos se detallan a continuación para mejorar la lectura e interpretación de los resultados: grado de colaboración de los participantes (COLAB); nivel de motivación del alumnado (MOTIV); rol desempeñado dentro de un enfoque activo (ROLAC); grado de interacción docente-discente (INTRA); grado de interacción discente-discente (INTRB); trabajo de la competencia digital del alumnado (TRACD); nivel de consecución de los resultados de aprendizaje (RESUL).

El análisis descriptivo se llevó a cabo mediante los resultados obtenidos a nivel frecuencial y porcentual, además del empleo de la media (M), la desviación típica (DT), el coeficiente de apuntamiento de Fisher (CAF) y el coeficiente de asimetría de Pearson $(C A P)$. El análisis comparativo entre el grupo experimental y el grupo de control se efectuó mediante la prueba $t$-Student, además de emplearse la $d$ de Cohen y la correlación biserial $(r)$ para determinar el tamaño del efecto. Se ha considerado un $p<0.05$ como valor de significancia estándar (Greenland et al., 2016; Wasserstein y Lazar, 2016). El programa empleado para el análisis estadístico de los datos fue el Statistical Package for the Social Sciences (SPSS) en su versión 24 para Windows.

\section{RESULTADOS}

La Tabla 2 muestra los resultados obtenidos para las variables de estudio en el grupo control, es decir, el grupo muestral que ha desarrollado el componente práctico del proceso de enseñanza y aprendizaje sin la inclusión de la radio y la televisión como canales de aprendizaje. Los resultados reflejan un nivel medio-bajo en las variables analizadas ( $\mathrm{M}_{\text {totalizada }}=2.3$ ). La variable relacionada con el trabajo de la competencia digital es la que ha obtenido una puntuación menor, mientras que la relacionada con los resultados de aprendizaje ha obtenido la puntuación más alta.

Tabla 2: Resultados obtenidos para las variables de estudio en el grupo control

\begin{tabular}{|l|l|l|l|l|l|l|l|l|}
\hline \multirow{2}{*}{ Variables } & \multicolumn{3}{|c|}{ Escala Likert $n(\%)$} & \multicolumn{3}{c|}{ Parámetros } \\
\cline { 2 - 9 } & Nada & Poco & Bastante & Totalmente & M & DT & CAP & CA $_{F}$ \\
\hline COLAB & $6(20)$ & $15(50)$ & $7(23.3)$ & $2(6.7)$ & 2.16 & 0.819 & 1.422 & -0.263 \\
\hline MOTIV & $7(23.3)$ & $14(46.7)$ & $7(21.7)$ & $2(23.3)$ & 2.1 & 0.851 & 1.293 & -0.368 \\
\hline ROLAC & $5(16.7)$ & $7(23.3)$ & $14(46.7)$ & $4(13.3)$ & 2.52 & 0.921 & 1.645 & -0.815 \\
\hline INTRA & $5(16.7)$ & $9(30)$ & $13(43.3)$ & $3(10)$ & 2.48 & 0.884 & 1.676 & -0.743 \\
\hline INTRB & $6(20)$ & $13(43.3)$ & $9(30)$ & $2(6.67)$ & 2.23 & 0.778 & 1.397 & -0.672 \\
\hline RESUL & $2(5)$ & $8(28.3)$ & $18(60)$ & $2(6.7)$ & 2.67 & 0.671 & 2.511 & 0.311 \\
\hline TRACD & $6(29)$ & $22(73.3)$ & $1(3.3)$ & $1(3.3)$ & 1.88 & 0.551 & 1.604 & 2.816 \\
\hline Total & $37(17.6)$ & $88(41.9)$ & $69(32.9)$ & $16(7.62)$ & 2.3 & 0.851 & 1.503 & -0.613 \\
\hline
\end{tabular}

Por otro lado, la Tabla 3 muestra los resultados obtenidos para las variables de estudio en el grupo experimental, es decir, el grupo muestral que ha desarrollado las prácticas de la asignatura con base en un modelo pedagógico retroinnovador en el que la radio y la televisión se constituyen como canales de aprendizaje. Los resultados reflejan un nivel medio-alto en las variables analizadas $\left(\mathrm{M}_{\text {totalizada }}=2.95\right)$. La variable relacionada con el trabajo de la competencia digital ha obtenido la puntuación más alta, mientras que las relacionadas con la interacción docente-discente y los resultados de aprendizaje han obtenido las puntuaciones más bajas.

Tabla 3: Resultados obtenidos para las variables de estudio en el grupo experimental

\begin{tabular}{|l|l|l|l|l|l|l|l|l|}
\hline \multirow{2}{*}{ Variables } & \multicolumn{3}{|c|}{ Escala Likert $n(\%)$} & \multicolumn{3}{c|}{ Parámetros } \\
\cline { 2 - 9 } & Nada & Poco & Bastante & Totalmente & M & DT & CAP & CA $F$ \\
\hline COLAB & $4(13.3)$ & $6(20)$ & $12(40)$ & $8(26.7)$ & 2.82 & 0.991 & 1.832 & -0.829 \\
\hline MOTIV & $2(6.7)$ & $4(13.3)$ & $11(36.7)$ & $13(43.3)$ & 3.2 & 0.891 & 2.469 & 0.159 \\
\hline ROLAC & $3(10)$ & $3(10)$ & $17(56,7)$ & $7(23.3)$ & 2.9 & 0.888 & 2.137 & 0.088 \\
\hline INTRA & $4(13.3)$ & $7(21.7)$ & $16(53.3)$ & $3(11.7)$ & 2.73 & 0.855 & 1.908 & -0.387 \\
\hline INTRB & $4(13.3)$ & $5(16.7)$ & $12(40)$ & $9(30)$ & 2.87 & 0.911 & 1.883 & -0.711 \\
\hline RESUL & $1(3.33)$ & $8(26,7)$ & $19(63.3)$ & $2(6,7)$ & 2.73 & 0.679 & 2.549 & 0.469 \\
\hline TRACD & $0(0)$ & $0(0)$ & $13(43.3)$ & $17(56.7)$ & 3.55 & 0.529 & 4.811 & -1.002 \\
\hline Total & $18(8.57)$ & $33(15.7)$ & $100(47.6)$ & $59(28.1)$ & 2.95 & 0.896 & 2.184 & -0.209 \\
\hline
\end{tabular}

Con base en los resultados obtenidos en el análisis cuantitativo de las variables formuladas (Fig. 2), a continuación, se expone la comparación de medias entre los grupos de estudio. Los resultados reflejan que las medias para el grupo experimental son superiores a las del grupo control en todas las variables. 


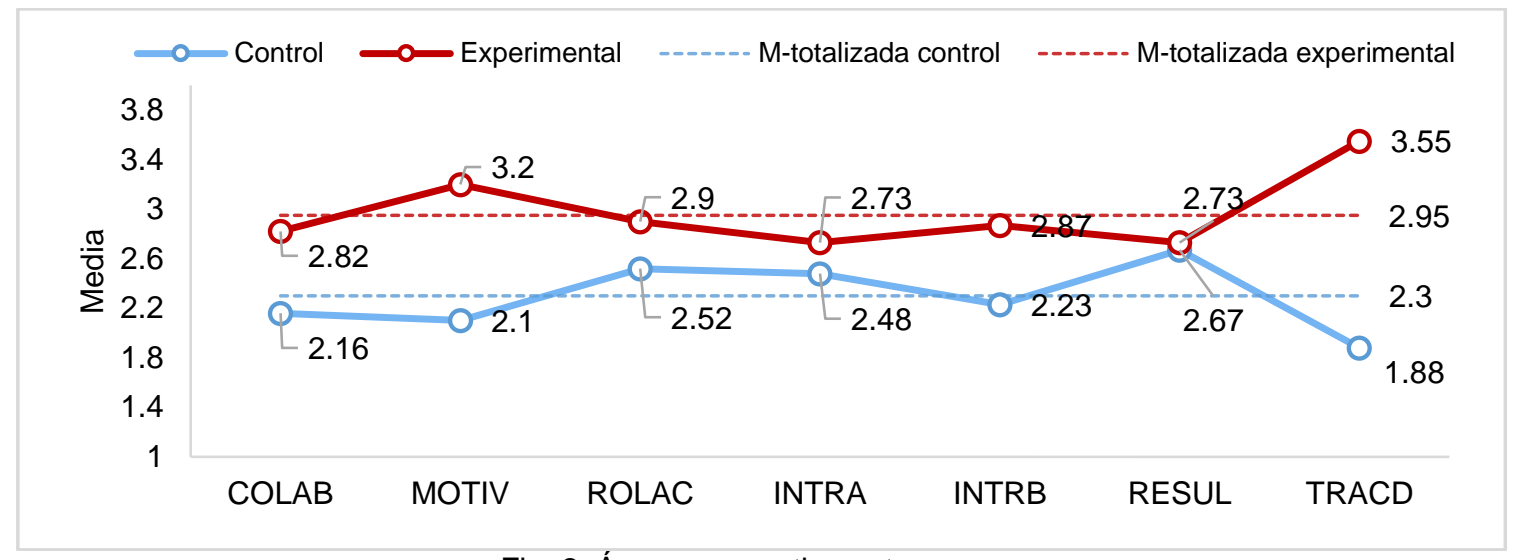

Fig. 2: Área comparativa entre grupos

Por último, la Tabla 4 muestra los resultados obtenidos para el análisis de la significancia entre el grupo control y el grupo experimental. Se han obtenido diferencias estadísticamente significativas $(p<0.05)$ en las variables relacionadas con la colaboración, la interacción discente-discente, la motivación y el trabajo de la competencia digital, siendo estas dos últimas en las que se ha obtenido una mayor fuerza de asociación tras el análisis del tamaño del efecto obtenido con los estadísticos $d$ de Cohen y la correlación biserial. No se han obtenido resultados estadísticamente significativos en el rol activo del discente, la interacción docente-discente y en los resultados de aprendizaje.

Tabla 4: Estudio del valor de independencia de los grupos muestrales

\begin{tabular}{|l|l|l|l|l|l|l|l|}
\hline \multirow{2}{*}{ Variables } & \multicolumn{2}{|c|}{ Grupo, M (DT) } & \multirow{2}{*}{$\mathrm{M}_{1}-\mathrm{M}_{2}$} & \multicolumn{2}{c|}{ Prueba $t$-Student } & \multirow{2}{*}{$-d$} & \multirow{2}{*}{$-r$} \\
\cline { 2 - 3 } & Control & Experimental & & $t(58)$ & $p$-valor & & \\
\hline COLAB & $2.16(0.82)$ & $2.82(0.99)$ & -0.66 & -2.73 & 0.008 & 0.726 & 0.341 \\
\hline MOTIV & $2.1(0.85)$ & $3.2(0.89)$ & -1.1 & -4.56 & $<0.001$ & 1.263 & 0.534 \\
\hline ROLAC & $2.52(0.92)$ & $2.9(0.89)$ & -0.38 & -1.77 & 0.082 & 0.419 & 0.205 \\
\hline INTRA & $2.48(0.88)$ & $2.73(0.86)$ & -0.25 & -0.65 & 0.517 & 0.287 & 0.142 \\
\hline INTRB & $2.23(0.78)$ & $2.87(0.91)$ & -0.64 & 2.17 & 0.034 & 0.755 & 0.353 \\
\hline RESUL & $2.67(0.67)$ & $2.73(0.68)$ & -0.06 & 0.38 & 0.704 & 0.088 & 0.044 \\
\hline TRACD & $1.88(0.55)$ & $3.55(0.53)$ & -1.67 & 3.78 & $<0.001$ & 3.092 & 0.839 \\
\hline
\end{tabular}

\section{DISCUSIÓN}

Las metodologías asociadas a la innovación deben formar parte inexorable de la práctica educativa diaria y deben estar sustentadas en la utilización de los recursos didácticos y tecnológicos que se encuentren a su alcance. Este hecho se encuentra ampliamente recogido en la literatura científica y ha sido abordado por multitud de autores. De esta forma, el presente apartado intenta abordar una comparativa entre los resultados obtenidos en el presente estudio y aquellos obtenidos por otras investigaciones de corte similar.

Resulta pertinente destacar que -derivado del análisis bibliográfico sobre el estado del arte- no ha sido posible encontrar estudios específicos que combinen el modelo flipped learning y la retroinnovación. De esta forma, el presente estudio se erige como un importante punto de partida para continuar la senda investigativa al respecto y poder enriquecer y dinamizar el proceso de enseñanza y aprendizaje. A pesar de ello, sí es posible comparar los resultados obtenidos en esta experimentación con otros de corte similar que -de manera independiente- tratan metodologías retroinnovadoras e innovadoras. En esta tipología de estudios, los resultados de los alumnos mejoran de forma ostensible tras la aplicación de un enfoque basado en el flipped learning, como revela la literatura reciente (Hinojo et al., 2018). Asimismo, se consigue un mejor clima de aula como consecuencia del aumento de la colaboración entre iguales y las relaciones con los docentes, como hallaron Hwang et al. (2015).

En concordancia con lo expuesto por Tourón y Santiago (2015), se ha obtenido una mejor autorregulación del aprendizaje por parte de los discentes, siendo destacable la mejora del proceso en el campo de la participación, la colaboración y la motivación, hallazgos que concuerdan con lo alcanzado por Tse et al., (2019). Por tanto, se han encontrado resultados similares a los obtenidos en la literatura en lo concerniente a la mejora de la socialización y de los resultados (MacLeod et al., 2017), la valoración positiva de la propuesta metodológica, la aceptación por parte del estudiantado (Lee, Park y Davis, 2018) y la inversión real de los tiempos y los espacios de aprendizaje (Boelens et al., 2018; Long, Cummins y Waugh, 2017).

El presente estudio ha analizado las diferencias entre los resultados de la aplicación de un método de enseñanza tradicional y de una metodología flipped learning complementada con un enfoque retroinnovador 
-tomando la radio y la televisión como canales de aprendizaje-. El alumnado que ha seguido un método de enseñanza y aprendizaje tradicional ha obtenido resultados inferiores en la totalidad de las variables propuestas para el estudio. De esta forma, ha obtenido peores resultados en aspectos relacionados con el grado de colaboración, el nivel de motivación, el rol desempeñado dentro de un enfoque activo, el grado de interacción docente-discente y discente-discente, el trabajo de la competencia digital y el nivel de consecución de los resultados de aprendizaje. Por contra, el alumnado que ha seguido una metodología novedosa fundamentada en el flipped learning y apoyada en medios retroinnovadores ha obtenido resultados superiores en todos los aspectos mencionados anteriormente, destacando -especialmente- el trabajo de la competencia digital. A pesar de que los resultados del modelo pedagógico retroinnovador son superiores en todas las variables analizadas con respecto al método de enseñanza tradicional, las diferencias estadísticamente significativas se han obtenido en los aspectos relacionados con la colaboración, la interacción discentediscente, la motivación y el trabajo de la competencia digital.

En definitiva, la presente investigación se configura como un estudio exploratorio que abre el camino de nuevas investigaciones que reflexionen sobre cómo afecta la retroinnovación dentro del flipped learning a cada una de las variables aquí analizadas. Como transferencia a la práctica de esta experiencia llevada a cabo en estudiantes de Educación Superior, cabe resaltar que puede ser extrapolada y adaptada a cualquier etapa educativa, adecuando tanto los espacios como los tiempos, así como teniendo en cuenta la competencia digital que poseen cada uno de los participantes que intervengan en la experimentación. Por otro lado, las limitaciones de este estudio se han centrado en los problemas que han tenido algunos estudiantes para acceder a los contenidos debido a problemas de conectividad y en el procesamiento de los materiales audiovisuales en sus dispositivos móviles, así como en el déficit de competencia digital que han presentado algunos participantes, lo cual ha dificultado el normal transcurso de la experiencia.

\section{CONCLUSIONES}

De acuerdo al trabajo presentado y a los resultados obtenidos, se pueden plantear las siguientes conclusiones principales: 1.- El contexto educativo actual ha provocado que se reduzca el uso de medios de comunicación clásicos como la radio y la televisión dentro del proceso de enseñanza y aprendizaje, de manera que su empleo pueda considerarse como una acción pedagógica retroinnovadora; 2.- En comparación con la metodología tradicional, la combinación del modelo flipped learning y de herramientas retroinnovadoras como la radio y la televisión contribuyen a potenciar en el alumnado el grado de colaboración, el nivel de motivación, el rol activo, el grado de interacción docente-discente y discente-discente, el trabajo de la competencia digital y el nivel de consecución de los resultados de aprendizaje; y 3.- La utilización de la radio y la televisión -junto con la tecnología digital actual-siguen siendo canales óptimos que se complementan para transmitir no solo información sino -además- generar y construir las ramas del conocimiento desde una perspectiva mediática y comunicativa, es decir, fomentando la inclusión de la educomunicación en el entorno académico.

\section{REFERENCIAS}

Area, M., Hernández, V., y Sosa, J.J., Modelos de integración didáctica de las TIC en el aula, http://doi.org/10.3916/C472016-08, Comunicar: Revista científica iberoamericana de comunicación y educación, 24(47), 79-87, (2016)

Basantes, A.V., Naranjo, M.E., Gallegos, M.C., y Benítez, N.M., Los dispositivos móviles en el proceso de aprendizaje de la Facultad de Educación Ciencia y Tecnología de la Universidad Técnica del Norte de Ecuador, http://doi.org/10.4067/S0718-50062017000200009, Formación Universitaria, 10(2), 79-88 (2017)

Bergmann, J., y Sams, A., Flip Your Classroom: Reach every student in every class every day, $1^{\text {a }}$ edición, 33-45. International Society for Technology in Education, Washington D. C., Estados Unidos (2012)

Boelens, R., Voet, M., y De Wever, B., The design of blended learning in response to student diversity in higher education: Instructors' views and use of differentiated instruction in blended learning, http://doi.org/10.1016/j.compedu.2018.02.009, Computers \& Education, 120, 197-212 (2018)

Cabero, J., y Barroso, J., Los escenarios tecnológicos en Realidad Aumentada (RA): posibilidades educativas en estudios universitarios, http://doi.org/10.17811/rifie.47.3.2018.327-336, Aula Abierta, 47(3), 327-336, (2018)

Cabero, J., La formación en la era digital: Ambientes enriquecidos por la tecnología, Revista Gestión de la Innovación en Educación Superior, 2(2), 41-64 (2017)

El Miedany, Y., Flipped learning; en The flipped classroom: Practice and practices in higher education de C. Reidsema, L. Kavanagh, R. Hadgraft y N. Smith, Spinger, pp 285-303, Nueva York, United State (2019)

Escobar, J., y Cuervo, A., Validez de contenido y juicio de expertos: una aproximación a su utilización, Avances en medición, 6(1), 27-36 (2008)

Froehlich, D.E., Non-technological learning environments in a technological world: Flipping comes to the aid, http://doi.org/10.7821/naer.2018.7.304, Journal of New Approaches in Educational Research, 7(2), 94-99 (2018) 
Fuentes, A., y López, J., TIC-TAC: Las tecnologías de la información y la comunicación en la era del acceso. Una experiencia en la educación superior, en Tendencias nacionales e internacionales en organización educativa: entre la estabilidad y el cambio de I. del Arco y P. Silva, Wolters Kluwer, pp 712-727, Madrid, España (2018)

Fuentes, A., López, J., y Pozo, S., Analysis of the Digital Teaching Competence: Key Factor in the Performance of Active Pedagogies with Augmented Reality, http://doi.org/10.15366/reice2019.17.2.002, Revista Iberoamericana sobre calidad, eficacia y cambio en educación, 17(2), 27-42, (2019)

Garrote, D., Garrote, C., y Jiménez, S., Factores influyentes en motivación y estrategias de aprendizaje en alumnos de grado, http://doi.org/10.15366/reice2016.14.2.002, Revista Iberoamericana sobre Calidad, Eficacia y Cambio en Educación, 14(2), 31-44 (2016)

Greenland, S., Senn, S.J., y otros cinco autores, Statistical tests, P values, confidence intervals, and power: a guide to misinterpretations, http://doi.org/10.1007/s10654-016-0149-3, European Journal of Epidemiology, 31(4), 337-350 (2016)

He, W., Holton, A., Farkas, G., y Warschauer, M., The effects of flipped instruction on out-of-class study time, exam performance, and student perceptions, http://doi.org/10.1016/j.learninstruc.2016.07.001, Learning and Instruction, 45, 61$71(2016)$

Hernández, R., Fernández, C., y Baptista, M.P., Metodología de la investigación, McGraw Hill, Madrid, España (2014)

Hinojo, F.J., Mingorance, A.C., y otros tres autores, Incidence of the Flipped Classroom in the Physical Education Students' Academic Performance in University Contexts, http://doi.org/10.3390/su10051334, Sustainability, 10(5), 1-13 (2018)

Hwang, G.J., Lai, C.L., y Wang, S.Y., Seamless flipped learning: a mobile technology-enhanced flipped classroom with effective learning strategies, http://doi.org/10.1007/s40692-015-0043-0, Journal of Computers in Education, 2(4), 449-473 (2015)

Lee, J., Park, T., y Davis, R.O., What affects learner engagement in flipped learning and what predicts its outcomes?, http://doi.org/10.1111/bjet.12717, British Journal of Educational Technology, 1(1), 1-18 (2018)

Long, T., Cummins, J., y Waugh, M., Use of the flipped classroom instructional model in higher education: instructors' perspectives, http://doi.org/10.1007/s12528-016-9119-8, Journal of Computing in Higher Education, 29(2), 179-200 (2017)

López, J., Pozo, S., Fuentes, A., y López, J.A., Creación de contenidos y flipped learning: un binomio necesario para la educación del nuevo milenio, http://doi.org/10.22550/REP77-3-2019-07, Revista española de pedagogía, 77(274), 535555 (2019)

MacLeod, J., Yang, H.H., Zhu, S., y Shi, Y., Technological factors and student-to-student connected classroom climate in cloud classrooms, http://doi.org/10.1177/0735633117733999, Journal of Educational Computing Research, 56(6), 826847 (2017)

Martín, E., Marta, C., y González, P., La radio universitaria en su dimensión formativa: Estudio de caso de Radio Unizar, http://doi.org/10.14198/MEDCOM2018.9.1.3, Revista Mediterránea de Comunicación, 9(1), 49-68 (2018)

McMillan, J., y Schumacher, S., Investigación educativa, 1a edición, 51-57. Pearson, Madrid, España (2005)

Mehandru, N., y Koenig, A., Icts, Social Media, \& the Future of Human Rights, Duke Law \& Technology Review, 17(1), 129-145 (2019)

Pascual, M.A., Ortega, J.A., Pérez, M., y Fombona, J., Competencias Digitales en los Estudiantes del Grado de Maestro de Educación Primaria. El caso de tres Universidades Españolas, http://dx.doi.org/10.4067/S0718-50062019000600141, Formación Universitaria, 12(6), 141-150 (2019)

Rodas, B., y Aveiga, A., Influencia de la Radio con un Enfoque Educomunicativo para la Formación Ciudadana, http://doi.org/10.33890/innova.v2.n8.1.2017.367, Innova Research Journal, 2(8), 224-236 (2017)

Rodríguez, A.M., Romero, J.M., y Agreda, M., Impact of ICT on the teaching of Physical Education: a bibliometric research study, http://doi.org/10481/53211, Education, Sport, Health and Physical Activity, 3(1), 1-14, (2019)

Rodríguez, N., Diseños experimentales en educación, Revista de Pedagogía, 32(91), 147-158 (2011)

Soberanes, A., Castillo, J., y Peña, A., Uso de recursos educativos abiertos en educación superior, Universidad \& Ciencia, 6(1), 124-137 (2017)

Tourón, J., y Santiago, R., El modelo Flipped learning y el desarrollo del talento en la escuela, Revista de educación, (368), 196-231 (2015)

Tse, W.S., Choi, L.Y., y Tang, W.S., Effects of video-based flipped class instruction on subject reading motivation, http://doi.org/10.1111/bjet.12569, British Journal of Educational Technology, 50(1), 385-398 (2019)

Villareal, S., García, J., Hernández, H., y Steffens, E., Competencias Docentes y Transformaciones en la Educación en la Era Digital, http://doi.org/10.4067/S0718-50062019000600003, Formación Universitaria, 12(6), 3-14 (2019)

Wasserstein, R.L., y Lazar, N.A., The ASA's statement on p-values: context, process, and purpose, http://doi.org/10.1080/00031305.2016.1154108, The American Statistician, 70(2), 129-133 (2016) 